\title{
Universiteit
}

Leiden

The Netherlands

\section{Electron induced ortho-meta isomerization of single molecules}

Simic-Milosevic, V.; Mehlhorn, M.; Rieder, K.H.; Meyer, J.; Morgenstern, K.

\section{Citation}

Simic-Milosevic, V., Mehlhorn, M., Rieder, K. H., Meyer, J., \& Morgenstern, K. (2007). Electron induced ortho-meta isomerization of single molecules. Physical Review Letters, 98(11), 116102. doi:10.1103/PhysRevLett.98.116102

Version: $\quad$ Not Applicable (or Unknown)

License: $\quad$ Leiden University Non-exclusive license

Downloaded from: https:/hdl.handle.net/1887/64307

Note: To cite this publication please use the final published version (if applicable). 


\title{
Electron Induced Ortho-Meta Isomerization of Single Molecules
}

\author{
V. Simic-Milosevic, ${ }^{1, *}$ M. Mehlhorn, ${ }^{1}$ K.-H. Rieder, ${ }^{1}$ J. Meyer, ${ }^{2}$ and K. Morgenstern ${ }^{2}$ \\ ${ }^{1}$ Institut für Experimentalphysik, Freie Universität Berlin, Arnimalle 14, 14195 Berlin, Germany \\ ${ }^{2}$ Institut für Festkörperphysik, Leibniz Universität, Appelstrasse 2, 30167 Hannover, Germany
}

(Received 5 May 2006; published 12 March 2007)

\begin{abstract}
A scanning tunneling microscope operating at $5 \mathrm{~K}$ is used to induce the isomerization of single chloronitrobenzene molecules on $\mathrm{Cu}(111)$ and verify the reaction. The threshold voltage of $(227 \pm 7) \mathrm{mV}$ for this reaction is explained based on electron-induced vibrational heating. We propose that the isomerization is initiated by simultaneous excitation of two vibrational molecular modes via inelastically tunneling electrons. This excitation results in a shift of the distribution probability of chlorine and hydrogen positions, which facilitates their mutual exchange.
\end{abstract}

DOI: 10.1103/PhysRevLett.98.116102

Two decades after the development of the scanning tunneling microscope (STM) allowing imaging of individual adsorbates, the scientific challenges have shifted to the precise STM manipulation of atoms and the control of reactions at a single molecule level [1]. Single molecule manipulations of importance to modern chemistry and material science, such as rotation [2], diffusion [3-5], and desorption [5], were investigated. In these studies extrinsic molecular bonds were modified by inelastically tunneling electrons. Some attention was also paid to the investigation of chemical reactions as dissociation of intrinsic molecular bonds [6-8] as well as the formation of bonds between two molecule fragments [7].

Novel reaction types in manipulation concerning unimolecular reactions are still sparse. A reversible conformational change of a metalloporphyrin was explained by electrostatic interaction [9]. Furthermore, a reversible change between cis- and trans- structures, induced by inelastically tunneling electrons, was demonstrated for single azobenzene derivates [10]. This process is known in chemistry as isomerization, a change of one molecular structure into another one with the same component atoms. Since isomers usually differ significantly in physical, chemical, and biological properties, isomerization opens a way for manipulating molecular properties.

Generally, isomers always have the same formula, but concerning the connectivity, two types of isomers are discerned. While constitutional isomers differ in connectivity, stereoisomers have the same connectivity and differ only in the arrangement of their atoms in space. While in [10] a stereoisomerization was demonstrated, we present in this Letter the hitherto not-achieved electron-induced constitutional isomerization of single chloronitrobenzene $\left(\mathrm{C}_{6} \mathrm{H}_{4} \mathrm{ClNO}_{2}\right)$ molecules on $\mathrm{Cu}(111)$. This isomerization, which has so far only been investigated in the liquid phase with a $\mathrm{H}_{2} \mathrm{SO}_{4}$ catalyst, occurs via the SN1 mechanism (nucleophilic substitution) [11].

The isomerization of $\mathrm{C}_{6} \mathrm{H}_{4} \mathrm{ClNO}_{2}$ is based on the exchange of a chlorine atom with a hydrogen atom attached to two different carbon atoms of the benzene ring. Since hydrogen atoms are invisible in STM images, the isomer-
PACS numbers: 68.37.Ef, 68.43.Pq, 82.30.Qt, 82.37.Gk

ization is confirmed through the position change of the chlorine. A control experiment corroborates that the chlorine atom stays chemically bound to the molecule during the isomerization. We suggest an excitation mechanism based on the vibrational heating model [12,13] in which the isomerization reaction is induced by simultaneous excitation of two vibrational molecular modes.

The experiments are performed at $5 \mathrm{~K}$ with a custombuilt ultrahigh-vacuum low-temperature STM [14]. The $\mathrm{Cu}(111)$ sample was cleaned by repetitive cycles of $\mathrm{Ne}^{+}$sputtering and annealing at $870 \mathrm{~K}$. Metachloronitrobenzene $(m$-ClNB) molecules $(100 \%$ purity in chromatogram) were deposited within $30 \mathrm{~s}$ on $\mathrm{Cu}(111)$ at $17 \mathrm{~K}$. After this preparation both $m$-ClNB and ortho(o-ClNB) (ratio 10:1) are found in STM images [15]. This indicates the catalytic activity of the surface that leads to an isomerization of the molecule upon contact with the surface. All STM images presented in this Letter are recorded at a bias voltage of $100 \mathrm{mV}$ and a tunneling current of $50 \mathrm{pA}$. Vibrational modes of the molecules are calculated in the gas phase using density functional theory (DFT) as implemented in the quantum chemistry package GAUSSIAN03 [16] [DFT-GGA PW91 [17] functional for correlation and exchange, 6-31G $(d, p)$ basis set for $\mathrm{H}$, 6-311G $(d, p)$ for $\mathrm{C}, \mathrm{N}, \mathrm{O}$, and $\mathrm{Cl}]$.

A prerequisite for proving STM induced isomerization is the unambiguous identification of molecular parts in the STM images, i.e., submolecular resolution. The adsorption experiments of $\mathrm{ClNB}$ molecules on $\mathrm{Cu}(111)$ in connection with tight-binding calculations [18] facilitate the interpretation of the STM images in Fig. 1. For similar tunneling parameters the phenyl ring is imaged as a shallow depression, an ellipsoidal protrusion next to this depression corresponds to the nitro group, and the circular protrusion of smaller apparent height $(30 \mathrm{pm})$ corresponds to the chlorine atom. This protrusion appears for the majority of molecules in the STM images exactly in the direction of the $\mathrm{C}-\mathrm{Cl}$ bond. Thus, we are able to distinguish the different isomers through the relative position of the chlorine atom with respect to the nitro group (Fig. 1). 


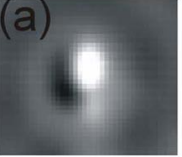

o-CINB-r

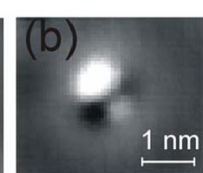

m-CINB-r

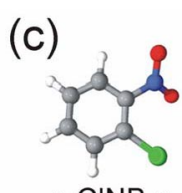

o-CINB-r (d)

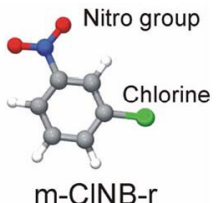

FIG. 1 (color online). STM images of (a) $o$-CINB- $r$ and (b) $m$ ClNB- $r$ isomers on $\mathrm{Cu}(111)$ with (c),(d) corresponding structural ball and stick models based on the optimized geometries as calculated by DFT. " $l$ " and " $r$ " denote that chlorine is adsorbed to the left-hand side or right-hand side of the nitro group.

Figure 2 shows the feasibility to isomerize a single CINB molecule. Shown are three manipulations out of a series of 60 performed on the same molecule. In the experiment, the molecule is first imaged with low bias and current. Figure 2(a) (left-hand side) shows $m$-CINB. Then the tip is moved above the protrusion that characterizes the chlorine. There the feedback loop is switched off, bias voltage and tunneling current are increased to $300 \mathrm{mV}$ and $130 \mathrm{pA}$, and held there for $12 \mathrm{~s}$, while recording the tunneling current. The drop in current at $\sim 8 \mathrm{~s}$ suggests a movement of the chlorine [19]. Indeed, the STM image in Fig. 2(a) (right-hand side) corresponds to $o$-ClNB; i.e., the chlorine moved to a new binding site within the molecule. The nitro group is always unaffected by the manipulation.

With the manipulation procedure described above it is also possible to manipulate the chlorine atom from different positions back to the meta- $l$ constitution [Figs. 2(b) and 2(c)]. The manipulation in Fig. 2(b) is the reverse isomerization compared to Fig. 2(a), while Fig. 2(c) illustrates the change from meta- $r$ to meta- $l$, i.e., a change in chirality.

(a)
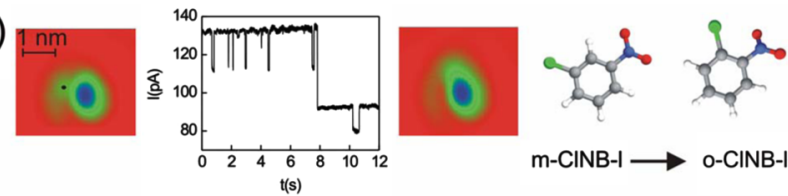

(b)
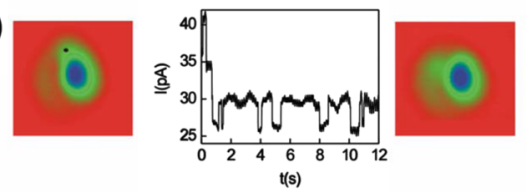

(c)
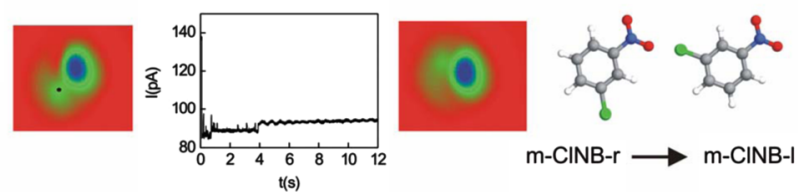

FIG. 2 (color online). Three manipulations performed on the same molecule: isomerization from (a) $m$-CINB- $l$ to $o$-CINB- $l$, (b) $o$-ClNB- $l$ to $m$-ClNB- $l$, (c) $m$-ClNB-r $r$ to $m$-ClNB- $l$. Ellipses correspond to nitro groups, circles represent chlorine atoms. Left STM images show molecule before and right STM images after manipulation. $300 \mathrm{meV}$ electrons were injected, where indicated by black dots. Jumps in $I-t$ curves during manipulation indicate position changes of chlorine [19]. On the right-hand side the isomerization is illustrated schematically.
The result of the isomerization is controllable by the excitation energy. Tunneling electrons with energies up to $300 \mathrm{meV}$ induce only isomerization between ortho- and meta- constitutions, while the chirality remains unaffected. The change of chirality between meta- $r$ and meta- $l$ constitutions is induced by tunneling electrons with energies starting from $300 \mathrm{meV}$. The isomerization between meta- $l$ and ortho- $r$ and between ortho- $l$ and ortho- $r$ constitutions is possible by dosing $500 \mathrm{meV}$ electrons. Para-constitution was never induced in any manipulation experiment.

In the following we elucidate the reaction mechanism. The low threshold barrier excludes a mechanism, in which chlorine and hydrogen are first detached from the molecule and then reattached to another position on the ring. Dissociation experiments on benzene on $\mathrm{Cu}(001)$ [20] and on phenyl residues on $\mathrm{Cu}(111)$ [21] showed that the $\mathrm{C}-\mathrm{H}$ bond can be broken only at tunneling voltages above $(2.9 \pm 0.1) \mathrm{V}$. Electron transmission spectroscopy measurements showed that the $\mathrm{C}-\mathrm{Cl}$ bond within a single chlorobenzene molecule in the gas phase can be broken after dosing $700 \mathrm{meV}$ electrons to the molecule [22], a value where dissociation is also possible here (see below). Thus a detachment-reattachment mechanism requires a minimum energy of approximately $3.6 \mathrm{eV}$, which is far above the energies used in our experiments. Electronic transition processes are also not suitable mechanisms for the isomerization, since the thresholds for the investigated unimolecular reaction are independent of bias polarity. Furthermore, the gap between the lowest unoccupied and highest occupied states of both CINB isomers in the gas phase is calculated to be $3.2 \mathrm{eV}$. This leaves electroninduced vibrational heating as the most obvious excitation mechanism behind the reaction $[12,13]$. To strengthen this statement with the aim of identifying the underlying excitation mechanism of this reaction, we investigate the change from ortho- $l$ to meta- $l$ and from ortho- $r$ to meta- $r$ constitution in greater detail by measuring the residence times that chlorine spends in different positions before isomerization within the molecule as a function of bias voltage $(180 \ldots .290 \mathrm{meV})$ and tunneling current (26...260 pA) (Fig. 3). Two different statistics are obtained, one for isomerization from $\mathrm{m}$ - to o-ClNB, the other one for the back reaction. All distributions of residence times within the current and voltage range probed follow a simple exponential law, allowing the determination of the time constants $\tau$ from exponential fits [Fig. 3(a)]. The observed linear dependence of time constants on the tunneling current [Fig. 3(a), inset] means that the isomerization is induced by a one electron process $[12,13]$.

The reaction yields per incident electron as a function of the manipulation voltage [Fig. 3(b)] increase at threshold voltages of $(229 \pm 7) \mathrm{mV}$ for the constitutional change from meta- into the ortho- and at $(224 \pm 7) \mathrm{mV}$ for the back reaction [23]. These values lie in the typical range of vibrational excitations. The dependence of the yield on voltage and current confirms that the reaction is induced by vibrational heating. 

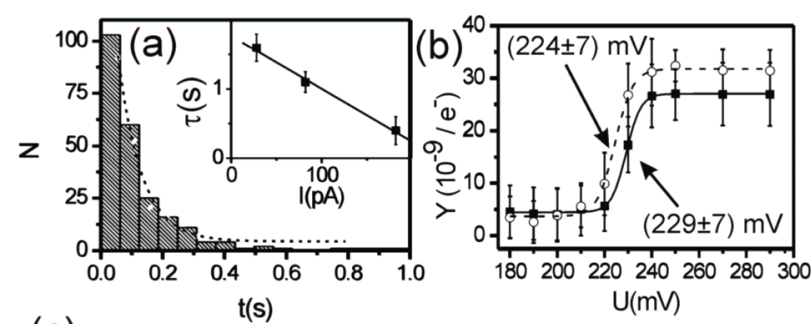

(c)

t(s)

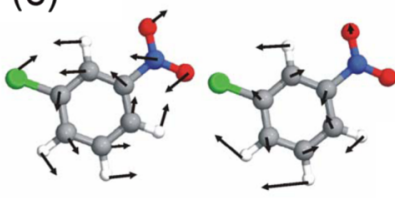

(I) $+($ II)

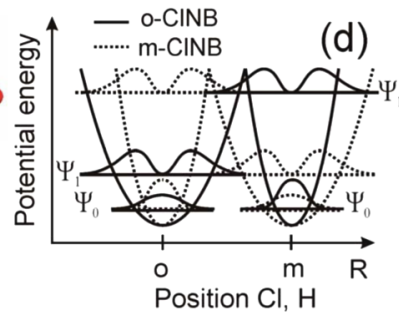

FIG. 3 (color online). (a) Histogram of time $t$ the chlorine spent in the meta- constitution before isomerizing to the orthoconstitution at fixed voltage $U=230 \mathrm{mV}$ and current $I=$ $260 \mathrm{pA}$. The dashed line represents an exponential fit. Inset (a): Time constants $\tau$ extracted from distributions as shown in main image recorded at $U=230 \mathrm{mV}$ as a function of current $I$. The solid line is a fit to the data corresponding to the power law $\tau^{-1} \propto I^{n}$, with $n=1.2 \pm 0.2$. (b) Yields $Y$ as a function of $U$ for isomerization of chlorine from meta- to ortho- constitution (black squares, fit: solid line) and from ortho- to meta- constitution (open circles, fit: dashed line). (c) Normal mode displacements for the vibrations (I) and (II) for $m$-CINB as calculated by GAUSSIAN03 represented by arrows. (d) Schematics of potential energy curves for chlorine (broad parabola) and hydrogen (narrow parabola) in-plane bending vibrations with states $n=0$ and $n=1$. Solid and dashed line wells correspond to a molecule being in ortho- and meta- constitution, respectively. The ground state $\Psi_{0}$ and excited $\Psi_{1}$ wave functions are shown. The reaction coordinate $R$ approximates the path between the chlorine and hydrogen atoms, along which they move for these particular vibrations; note that the parabolas should be orthogonal.

Because of a lack in experimentally recorded vibrational spectra of ClNB isomers, we have calculated the vibrational modes of chlorobenzene, nitrobenzene, $m$-CINB, and $o$-ClNB in the gas phase by DFT calculations in order to identify the vibrations involved in the reaction. The first two molecules serve to check the validity of the method by comparison to experimental data both in the gas phase and adsorbed on surfaces [24,25]. We find a mean square deviation between the literature data and our calculation for the 30 , respective 36 , modes of less than $2 \mathrm{meV}$ for vibrations within the energy range of interest and thus far below our experimental uncertainty $(7 \mathrm{meV})$. Comparison of our experimental data on surfaces to gas phase calculation is justified by a negligible shift of the vibrational spectra observed for benzene upon adsorption on $\mathrm{Al}(111)$ [26] and nitrobenzene on $\mathrm{Au}(111)$ [25].

The vibrations found in DFT show energies below $199 \mathrm{meV}$ and above $388 \mathrm{meV}$, but none at the experimentally determined threshold voltage [27]. Thus, we suggest that the reaction might be induced by simultaneous exci- tation of two vibrational modes [28], preferably those containing $\mathrm{C}-\mathrm{Cl}$ and $\mathrm{C}-\mathrm{H}$ in-plane bending motions that lead to an approach of the two exchanging atoms. Only two combinations of the 36 existing modes fulfill this requirement and have the right energy, both involving a mode with a $\mathrm{C}-\mathrm{Cl}$ in-plane bending and a $\mathrm{C}-\mathrm{C}$ stretch mode that involves an in-plane bending of the hydrogen atom (see Table I). Figure 3(c) depicts the normal modes of one of the combinations for the meta- isomer [27].

For visualization of the processes in a quantum mechanical interpretation, we sketch the normal modes for $\mathrm{C}-\mathrm{Cl}$ and $\mathrm{C}-\mathrm{H}$ in-plane bending vibrations as Eigenstates of a one-dimensional harmonic oscillator. Figure 3(d) shows schematically ground state potential energy curves for chlorine and hydrogen in meta- and ortho- position (solid line) and ortho- and meta- (dashed line), respectively. The reaction coordinate $R$ approximates the distance between the exchanging atoms. We qualitatively propose that there is enough overlap of the wave functions for the chlorine and hydrogen atoms to allow for isomerization in the first excited vibrational states, where the probability distribution of the atoms are enhanced along the connecting line.

An alternative explanation for the small activation barrier is that the chlorine atom is only physisorbed on the phenyl ring and not chemically bound to the corresponding carbon atom. In this case the result of the manipulation would not be an isomerization but a diffusion of the chlorine atom on the phenyl ring. A control experiment rules out this objection (Fig. 4). In this experiment, the chlorine atom is first detached from the phenyl ring by injecting $700 \mathrm{meV}$ tunneling electrons into it [Fig. 4(a)] [29]. By sliding the detached chlorine over the surface at a tunneling resistance of $R=10^{7} \Omega$, along the black arrow in the STM image in Fig. 4(b), the chlorine atom is now placed on top of the phenyl ring of the remaining nitrobenzene [Fig. 4(c)]. The line scan above the molecule (c) shows that the physisorbed chlorine atom has an apparent height of $80 \mathrm{pm}$, in sharp contrast to the apparent height of the chemically bound chlorine of only $30 \mathrm{pm}$ (a). During the isomerization experiments the apparent height of a chlorine is always approximately $30 \mathrm{pm}$ and chlorine is thus chemically bound to the molecule.

In conclusion, we have demonstrated the possibility to induce constitutional isomerization of single ClNB iso-

TABLE I. Theoretical vibrational energies that sum to the experimental threshold.

\begin{tabular}{lcc}
\hline \hline \multicolumn{1}{c}{ Vibrational mode } & $\begin{array}{c}\text { Energy } \\
\text { for } m \text { - isomer }\end{array}$ & $\begin{array}{c}\text { Energy } \\
\text { for } o \text { - isomer }\end{array}$ \\
\hline (I) C-Cl in-plane bending & $40 \mathrm{meV}$ & $38 \mathrm{meV}$ \\
(II) C-C stretch & $180 \mathrm{meV}$ & $180 \mathrm{meV}$ \\
Sum 1 & $220 \mathrm{meV}$ & $218 \mathrm{meV}$ \\
(III) C-NO 2 in-plane bending & $21 \mathrm{meV}$ & $24 \mathrm{meV}$ \\
(IV) C-C stretch & $199 \mathrm{meV}$ & $197 \mathrm{meV}$ \\
Sum 2 & $220 \mathrm{meV}$ & $221 \mathrm{meV}$ \\
\hline \hline
\end{tabular}



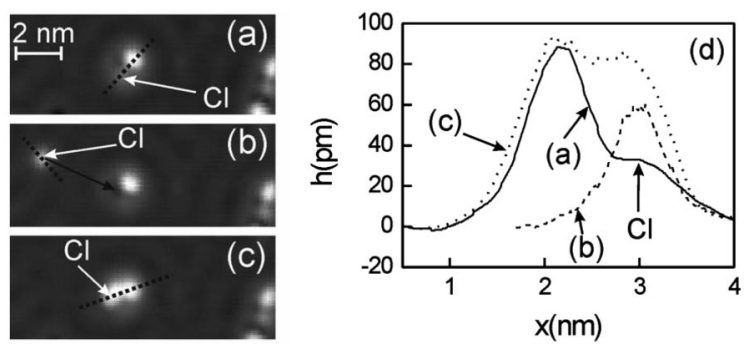

FIG. 4. Control experiment for unimolecular reaction. (a) STM image of $m$-CINB. STM electrons of $700 \mathrm{meV}$ are injected into chlorine atom resulting in (b). (b) STM image of chlorine detached and diffused from nitrobenzene rest. Black arrow indicates lateral manipulation in constant current mode at a tunneling resistance of $R=10^{7} \Omega$. (c) STM image of chlorine lying on top of the phenyl ring next to the nitro group. (d) Line scans as indicated in (a) to (c) by dashed lines.

mers on $\mathrm{Cu}(111)$ using electrons tunneling from the tip of a low-temperature STM. We suggest that this reversible isomerization is initiated by the simultaneous excitation of two vibrational modes that allow atoms attached to different carbon atoms to exchange their positions. However, the reaction mechanism can only be resolved by an advanced quantum chemical treatment of possible reaction paths, which we hope to induce with this article.

This model system serves to demonstrate that the isomerization without geometrical changes within a molecule adsorbed on a surface is feasible opening a way to manipulate molecule properties of single molecules, e.g., dipole moment or chirality, even for molecules within strict geometrical boundaries. The molecules investigated here possess a dipole moment in the gas phase, which is expected to be not quenched completely upon adsorption. The dipole moment strength depends on the constitution and is $(4.64 \pm 0.09) \mathrm{D}$ for $o-\mathrm{ClNB}$ and $(3.73 \pm 0.07) \mathrm{D}$ for $m$-CINB in the gas phase [30]. The corresponding results of our DFT calculations are 4.71 D and 3.67 D, respectively. Thus, ortho- meta- isomerization corresponds to an appreciable change in dipole moment. On the other hand, the achiral molecule becomes chiral due to a reconstruction of the molecule upon adsorption on $\mathrm{Cu}(111)$ (cf. [31]) and thus a change from meta- $l$ to meta- $r$ constitution corresponds to a change in chirality, an important property in biology. Finally, the reported isomerization reaction may be of interest to single molecular electronics. It has been shown that it is possible to prepare a rectifying junction based on the conductance switching of single molecules sandwiched between two electrodes upon field induced isomerization [32]. Recent results also show that isomerization can be used to manipulate the Kondo temperature in systems containing paramagnetic centers [33].

We gratefully acknowledge financial support provided by the Volkswagen Stiftung within the project "Catalysis on the nanoscale: Understanding and controlling molecular action on metallic surfaces."
*Email address: simic@ @hi-berlin.mpg.de

[1] W. Ho, J. Chem. Phys. 117, 11033 (2002).

[2] B. C. Stipe, M. A. Rezaei, and W. Ho, Phys. Rev. Lett. 81, 1263 (1998).

[3] T. Komeda et al., Science 295, 2055 (2002).

[4] K. Morgenstern and K. H. Rieder, J. Chem. Phys. 116, 5746 (2002).

[5] J. I. Pascual et al., Nature (London) 423, 525 (2003).

[6] B. C. Stipe et al., Phys. Rev. Lett. 78, 4410 (1997).

[7] S. W. Hla et al., Phys. Rev. Lett. 85, 2777 (2000).

[8] Y. Kim, T. Komeda, and M. Kawai, Phys. Rev. Lett. 89, 126104 (2002).

[9] X.H. Qiu, G. V. Nazin, and W. Ho, Phys. Rev. Lett. 93, 196806 (2004).

[10] J. Henzl et al., Angew. Chem., Int. 45, 603 (2006).

[11] J. Buddrus, Grundlagen der organischen Chemie, Berlin 1990.

[12] G. P. Salam, M. Persson, and R. E. Palmer, Phys. Rev. B 49, 10655 (1994).

[13] H. Ueba and B. N. J. Persson, Surf. Sci. 566, 1 (2004).

[14] M. Mehlhorn et al. (to be published).

[15] The para-CINB is very rarely observed. In a separate experiment where $p$-CINB was deposited we found that $p$-CINB dissociates upon adsorption (not shown).

[16] M. J. Frisch et al., GAUSSIAN 03, Revision C.02 (Gaussian, Inc., Wallingford CT, 2004).

[17] J.P. Perdew and Y. Wang, Phys. Rev. B 45, 13244 (1992).

[18] E. Niemi et al., J. Chem. Phys. 125, 184708 (2006).

[19] The two additional current values plateaus in the $I-t$ characteristic that might be related to the adsorption site change of the chlorine atom between neighboring bridge and hollow sites will be discussed elsewhere.

[20] L. J. Lauhon and W. Ho, J. Phys. Chem. A 104, 2463 (2000).

[21] S. W. Hla, G. Meyer, and K.-H. Rieder, Chem. Phys. Lett. 370, 431 (2003).

[22] P. D. Burrow, A. Modelli, and K. D. Jordan, Chem. Phys. Lett. 132, 441 (1986).

[23] Isomerization on $\operatorname{Ag}(111)$ and $\operatorname{Au}(111)$ showed similar thresholds.

[24] T. Skalycky et al., Phys. Chem. Chem. Phys. 4, 3583 (2002).

[25] D. Syomin, J. Wang, and B. Koel, Surf. Sci. 495, L827 (2001).

[26] R. Duschek et al., Chem. Phys. Lett. 318, 43 (2000).

[27] The complete results of the calculation can be found on http://www.fkp.uni-hannover.de/ morgenstern/ClNB/ index-ClNB.html.

[28] Many-orbital theory calculations showed that such a process is as likely as excitation to $n=2$ of either mode; N. Mingo and K. Makoshi, Surf. Sci. 438, 261 (1999).

[29] E. Illenberger, Chem. Rev. 92, 1589 (1992).

[30] D. R. Lide, Handbook of Chemistry and Physics (CRC Press, Boca Raton, 1999), 80th ed.

[31] J. Nieminen et al., Phys. Rev. B 72, 195421 (2005).

[32] A. Troisi and M. A. Ratner, J. Am. Chem. Soc. 124, 14528 (2002).

[33] V. Iancu, A. Deshpande, and S. W. Hla, Nano Lett. 6, 820 (2006). 